\title{
Ultrasound and acoustic analysis of sibilant fricatives in preadolescents and adults
}

\author{
Natalia Zharkova ${ }^{\text {a) }}$ \\ Clinical Audiology, Speech and Language Research Centre, Queen Margaret University, Edinburgh, \\ United Kingdom
}

(Received 16 July 2015; revised 18 March 2016; accepted 6 April 2016; published online 3 May 2016)

\begin{abstract}
This study describes the production of sibilant fricatives /s/ and $/ \int /$, comparing Scottish English speaking preadolescent children with adults. The materials were the sequences $/ \partial \mathrm{Ca} / \mathrm{and} / \mathrm{\partial C} / \mathrm{pro}-$ duced by 15 adults and 15 children aged between 10 and 12 years old. Quantitative analyses were carried out on both spectral information and on ultrasound imaging data on tongue shape, taken from nine successive time points during the fricative. The two groups of speakers were very similar to each other in the articulatory and acoustic characteristics distinguishing the two fricatives. Agerelated differences in the fricative centroid measure occurred at consonant-vowel boundaries, with lower values in the preadolescents. Within-speaker variability was mostly similar across age groups, with the exception of the fricative centroid for $/ \int /$, which was significantly more variable in preadolescents than in adults. Throughout the consonant duration, both groups consistently differentiated between the two consonants in both the fricative centroid and in one measure of tongue shape. (C) 2016 Acoustical Society of America. [http://dx.doi.org/10.1121/1.4947046]
\end{abstract}

[CYE]

Pages: 2342-2351

\section{INTRODUCTION}

Multiple studies have demonstrated that speech motor development is not yet complete by adolescence (e.g., Lee et al., 1999; Walsh and Smith, 2002; Koenig et al., 2008). In particular, it has been shown that aspects of the production of sibilant fricatives are still undergoing some development in early adolescence (Romeo et al., 2013). It is known that the extent of differentiation between $/ \mathrm{f} /$ and $/ \mathrm{s} /$ is smaller in 2-7-year-olds than in adults (Nittrouer et al., 1989; Li et al., 2009; Holliday et al., 2015), but it is not clear at what point children achieve consistent adult-like productions. The present study describes the production of sibilant fricatives in Scottish English speaking adults and preadolescents aged between 10 and 12 years old. Dynamic analyses during the fricative were carried out using acoustic data and direct information on tongue shape from ultrasound imaging. ${ }^{1}$ To the author's knowledge, there are currently no published studies based on both articulatory and acoustic data comparing dynamic properties of $/ \mathrm{s} /$ and $/ \int /$ production in adults and in children. The corpus used in the analyses was recorded for an articulatory-only study of lingual coarticulation, reported in Zharkova et al. (2014). That study found fine timing differences across age groups, with a later-than-adult onset of anticipatory vowel-on-consonant coarticulation in preadolescents for $/ \int /$, despite adult-like performance for /s/ on spatial and temporal measures of coarticulation. Zharkova et al. (2014) investigated the influence on the consonant from contrasting vowels, but did not address cross-consonant differences independent of these vowels. Documenting these differences is important for understanding the developmental pattern of the sibilant contrast, and it can help to identify

${ }^{a)}$ Electronic mail: nzharkova@qmu.ac.uk articulatory mechanisms responsible for any remaining immaturities in realising the contrast at preadolescent age. The cross-consonant differences and any age-related effects on them are the focus of the present study.

Articulatory measurements were based on ultrasound imaging including the extent of tongue "bunching" (in this paper, "bunching" refers to the tongue excursion in relation to the two ends of the imaged tongue curve). This was quantified using the Dorsum Excursion Index (DEI), which has been shown to reliably distinguish between some aspects of adult tongue shapes across consonants (Zharkova, 2013b), with larger values of the index corresponding to more bunched tongue shapes, and smaller values of the index representing flatter tongue shapes. The location of the most bunched part of the tongue in relation to the ends of the tongue curve was measured using the $\mathrm{LOC}_{\mathrm{a}-\mathrm{i}}$ index, which differentiates between tongue shapes with the most excursed point being further forward along the tongue curve, such as in [i], versus further back along the curve, such as in [a] (Zharkova et al., 2015). A number of acoustic measures were also made in the current study. The change in spectral properties during the consonant was measured using the fricative centroid (cf. Nittrouer et al., 1989; Li et al., 2009; Romeo et al., 2013). The frequency of the second formant was used to infer the relative length of the cavity behind the constriction (cf. McGowan and Nittrouer, 1988; Jongman et al., 2000; Li et al., 2009) at the consonant-vowel boundaries. The relationship between articulatory and acoustic measures was investigated, as well as within-speaker variability on all measures.

\section{METHOD}

The dataset used in this paper is described in more detail in Zharkova et al. (2014). The speakers were 15 typically 
developing preadolescents (six female) and 15 adults (12 female), all native speakers of Scottish Standard English. The mean age of the preadolescents was 11;2 ([years; months]), with the range $10 ; 0$ to $12 ; 4$. The mean age of the adults was 37 years with the range 18 to 58 years. The materials were the syllables $/ \int \mathrm{i} /, / \int \mathrm{a} /, / \mathrm{si} /$, and $/ \mathrm{sa} /$, embedded in the carrier phrase "It's a..., Pam." The sentences were presented to the participants on a computer screen (six repetitions of every target, in random order), and the target CV syllables were spelled as "she," "shah," "sea," and "sah," respectively. An Ultrasonix Sonix RP ultrasound scanner, with a C9-5/10 microconvex transducer, was used for recording tongue movements in the midsagittal plane. ARTICULATE ASSISTANT ADVANCED software (Articulate Instruments Ltd., 2012), running on a computer connected to the ultrasound scanner, was used for capturing ultrasound images (the frame rate for ultrasound was $100 \mathrm{~Hz}$ ) synchronized with the acoustic signal. The latter was recorded at $22050 \mathrm{~Hz}$ with an Audiotechnica AT803d microphone. Participants wore a headset which stabilized the ultrasound transducer in relation to the head.

The procedures of annotation, tongue curve fitting and normalization for time were carried out using ARTICULATE ASSISTANT ADVANCED. The consonant onset was defined as the onset of the frication noise in the acoustic signal. In several children, preaspirated tokens of the consonant were present; in such cases the onset of the consonant was located after the preaspiration interval, at the abrupt increase of the noise amplitude for the fricative. The consonant offset was defined as the onset of the periodic signal following the consonant. In each consonant token, tongue curves were traced for every ultrasound frame between frication onset and offset. The tracing was carried out automatically, with manual correction where necessary (more details on the manual correction procedure can be found in Zharkova et al., 2014). Tongue curves at nine equally spaced time points between consonant onset and offset (inclusive) were used for the articulatory analysis in this study.

The analyses focused on the two fricatives' differentiation in the two groups of speakers, and particularly, in view of the previous studies mentioned above, on any age-related differences in realising the /s/-/ $/$ / contrast. The articulatory indices used in this paper were chosen as they have the potential to quantify the differences in tongue shape between $/ \mathrm{s} /$ and $/ \mathrm{J} /$, which, in turn, may contribute to creating crossconsonant differences in the size of the cavities either side of the constriction. As these articulatory indices have not been previously used to quantify differences between postalveolar and alveolar fricatives, in this study the indices were subjected to correlations with well-established spectral measures, in order to establish whether articulatory and acoustic measures of the $/ \mathrm{s} /-/ \int /$ difference could be used interchangeably, and if there was any complementarity between the two.

The indices were calculated in $\mathrm{R}(\mathrm{R}$ Development Core Team, 2012) for each fricative token at every time point. The calculations of DEI are illustrated in Fig. 1(a) using two tongue curves from a preadolescent speaker, at mid-/s/ from /sa/ and at mid-/ $/$ / from $/ \int \mathrm{a} /$. In order to obtain the DEI value for a given tongue contour, a straight line is first traced between the two ends of the tongue curve (line $n$ ), and then a

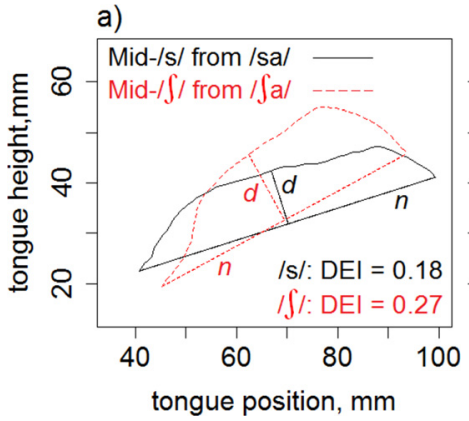

b)

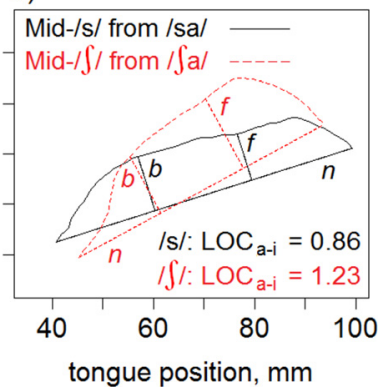

FIG. 1. (Color online) Example tongue curves for /s/ (solid) and / $/$ / (dashed) from a preadolescent speaker, illustrating the calculations of (a) DEI; (b) $\mathrm{LOC}_{\mathrm{a}-\mathrm{i}}$. The front of the tongue is on the right in all the figures.

perpendicular from its midpoint to the tongue curve is traced (line $d$ ). The DEI value is the ratio $d / n$, corresponding to the extent of tongue bunching. While the length of line $n$ in Fig. 1 (a) is longer for $/ \mathrm{s} /(61.5 \mathrm{~mm})$ than for $/ \mathrm{f} /(55.3 \mathrm{~mm})$, the perpendicular $d$ is shorter for $/ \mathrm{s} /(11.0 \mathrm{~mm})$ than for $/ \int /$ $(14.8 \mathrm{~mm})$, and the resulting DEI value is therefore larger for the postalveolar fricative $(0.27)$ than for the alveolar $(0.18)$.

The $\mathrm{LOC}_{\mathrm{a}-\mathrm{i}}$ index calculations are illustrated in Fig. 1(b), using the same two tongue curves as in Fig. 1(a). Using line $n$, perpendiculars are traced to the tongue curve from the points located at one third and two thirds of line $n$, starting from the front of the tongue (lines $f$ and $b$, respectively). The $\mathrm{LOC}_{\mathrm{a}-\mathrm{i}}$ value is the ratio $f / b$, representing the location of the most excursed part of the tongue relative to the rest of the curve. In Fig. 1(b), while line $f$ is longer than line $b$ for $/ \int /(15.7 \mathrm{~mm}$ versus $12.8 \mathrm{~mm}$, respectively), the opposite is the case for $/ \mathrm{s} /$ (9.6 mm versus $11.2 \mathrm{~mm}$, respectively), so the $\mathrm{LOC}_{\mathrm{a}-\mathrm{i}}$ value is larger for the postalveolar (1.23) than for the alveolar (0.86). Higher LOCa-i values mean that the bunching occurs in the more anterior part of the tongue, while lower LOCa-i values refer to those cases where the more posterior part of the tongue is bunched. The index does not provide information on the place of constriction relative to the palate, and "location of bunching" throughout the paper means relative location within the tongue curve. Therefore references to more anterior or more posterior tongue bunching in the paper describe tongue shapes, rather than the tongue position in the mouth.

Calculating both articulatory indices in this study required establishing the two ends of the tongue curve for each token. The ends of the curve were located at the intersection of the visible tongue contour and the shadows of the chin and of the hyoid bone. ${ }^{2}$ A potential methodological issue is that such indices might be affected by potential differences in the proportion of the visible tongue curve, and therefore in the location of the two curve ends, due to the tip or the back of the tongue not fully imaged with ultrasound. DEI has been previously demonstrated to be slightly affected by changes in the length of the imaged curve (Zharkova, 2013a), while $\mathrm{LOC}_{\mathrm{a}-\mathrm{i}}$ has been shown to be more resilient than DEI to potential changes in the length of the curve (Zharkova et al., 2015). In this study, there was a possibility that some of the tongue tip was missing for $/ \mathrm{s} /$, due to the air below it. However because this possibility applied to all speakers, it is unlikely that any tip imaging issues affected the results. 

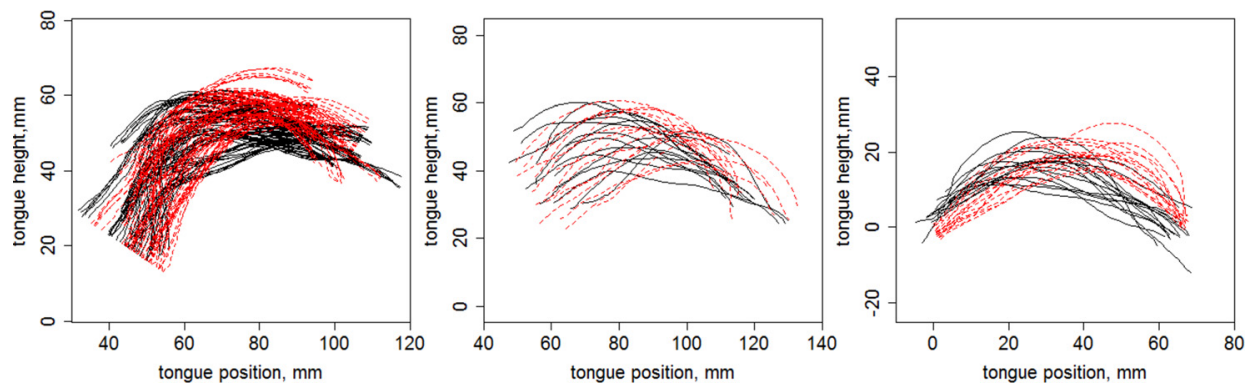

FIG. 2. (Color online) Illustration of the normalization process used for visual display. All three panels display tongue curves in all preadolescents at mid-/s/ from /sa/ (solid) and mid-/ / from / $\mathrm{a}$ / (dashed). The left panel shows "raw" data, i.e., there are six repetitions per speaker, and the curves are not shifted and not rotated. In the middle panel, each curve is an average per speaker, and all curves have been rotated. The right panel shows the average curves for each speaker after they have been shifted and the normalization factor has been applied.

Spectral analysis of the two fricatives was performed using R scripts described in Reidy (2013) and adapted by Romeo et al. (2013). An audio file containing each fricative token was band-pass filtered, with the low cut-off frequency of $300 \mathrm{~Hz}$ and the high cut-off frequency of $20000 \mathrm{~Hz}$. Multitaper spectra (e.g., Blacklock, 2004), with eight orthogonal tapers, were calculated for each of eight equal intervals between the fricative onset and offset. Similarly to Romeo et al. (2013), Koenig et al. (2013), and Shadle et al. (2014), no preemphasis was applied. Using the multitaper spectra, four spectral moments were calculated. The four moments were all correlated with each other, so only the results for the fricative centroid will be reported here.

F2 was measured at the onset and offset of the fricative in PRAAT (Boersma and Weenink, 2013), for each fricative token. The author visually examined the formant values using a wideband spectrogram, with superimposed automatically tracked formant contours (maximum formant: $5500 \mathrm{~Hz}$ for adult women and preadolescents, $5000 \mathrm{~Hz}$ for adult men; number of formants: 5; window length: $25 \mathrm{~ms}$ ). A PRAAT-derived formant value was accepted if it was located within the grey area for the formant on the spectrogram. If the PRAAT-derived value was visually outside the formant band on the spectrogram, then the formant value at that time point was located by hand at the centre of the formant on the spectrogram. In total, F2 values were located by hand for 329 tokens. Of those values, $8 \%$ selected randomly were assessed by another phonetician, with the mean difference of $78 \mathrm{~Hz}$ between the two assessors. Author-estimated values were included in further analyses.

To analyse variability, the coefficient of variation was calculated within speaker for all measures, separately for each fricative, vowel context and time in the consonant, by computing the ratio of the standard deviation to the mean. The coefficient of variation was chosen for analysing variability because it allows for normalization for any changes in the spread due to large changes in the mean over time (such as a noticeable increase in both DEI and $\mathrm{LOC}_{\mathrm{a}-\mathrm{i}}$ during the fricative preceding the high front vowel) or age-related differences in the mean (as was the case for F2 values). Additionally, following Romeo et al. (2013), discriminability between the two fricative categories was calculated for every measure, also within speaker, separately for each vowel context and time in the consonant. Discriminability was defined as the ratio of the difference between the mean values of the two fricatives (i.e., an indication of how far the fricatives are from each other) to the square root of the mean of the variances (i.e., a measure of the spread for the two fricatives taken together). This measure was chosen because if there was, as expected, a difference between the two fricatives in both age groups, it would make it possible to quantify the extent of separation between the two fricative categories and compare it across age groups.

\section{A. Tongue curve averaging and normalization for visual display}

In order to display average tongue movement patterns for each age group, shown in Figs. 3 and 4, it was necessary to normalize for vocal tract size and for ultrasound transducer orientation. This subsection describes the normalization procedures, and illustrations are provided in Fig. 2. For each speaker, tongue curves for the six repetitions of each consonant in each vowel context and at each time point were averaged in ARTICULATE ASSISTANT ADVANCED. Also within this software, these mean curves were rotated, to achieve the position where the curve for the onset of $/ \mathrm{J} /$ in the context of $/ \mathrm{a} /\left(\int_{\mathrm{a}} 1\right)$ had the same first and last Cartesian $y$ values (thus, in Figs. 3 and 4, only the $\int_{\mathrm{a}} 1$ curve has the same first and last $y$ values). The $x y$ values for the rotated curves were exported into text files, and further analyses were run in Microsoft EXCEL. For each age group, a speaker was identified who had the highest $x$ value for the $\int_{\mathrm{a}} 1$ curve. Within age group, consonant and time point, the highest $x$ value in
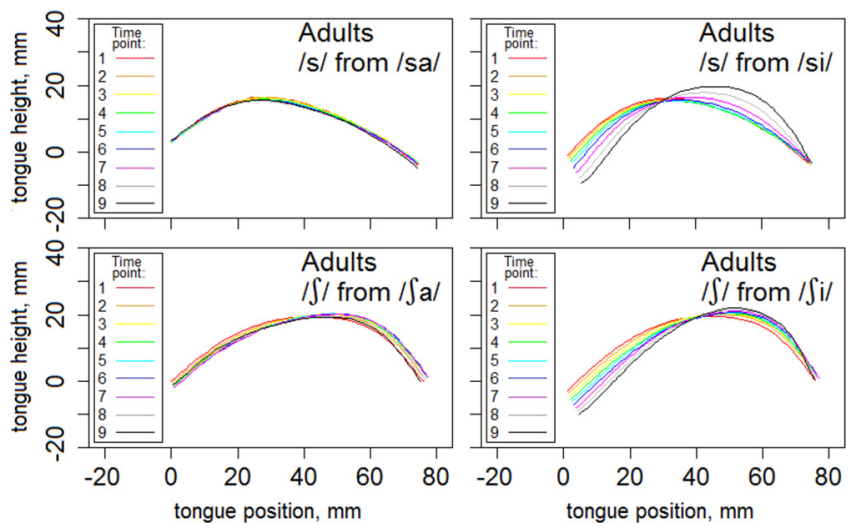

FIG. 3. (Color online) Tongue curves from nine tongue curves of $/ \mathrm{s} /$ and nine tongue curves of $/ \int /$, normalized and averaged across speakers, in the context of the two vowel contexts, produced by adults. 

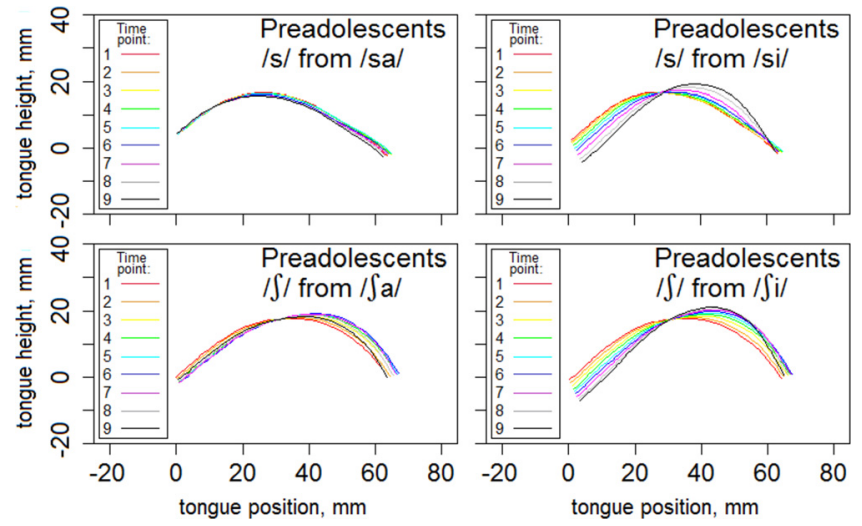

FIG. 4. (Color online) Tongue curves from nine tongue curves of $/ \mathrm{s} /$ and nine tongue curves of $/ \int /$, normalized and averaged across speakers, in the context of the two vowel contexts, produced by preadolescents.

the /a/ vowel context from this speaker, divided by the highest $x$ value in the /a/ vowel context for every speaker in the age group, constituted a normalization factor. As a result, absolute values on each graph in Figs. 3 and 4 correspond to the absolute dimensions, within age group, for the speaker who had the largest distance between the first and last $x$ values on the $\int_{\mathrm{a}} 1$ curve. For every speaker, each curve was shifted so that the first $x$ value, as well as the first $y$ value, of the $\int_{\mathrm{a}} 1$ curve for that speaker equaled an arbitrarily chosen value of zero. The normalization factor was then applied to every curve. Finally, the curves for each consonant, time point and age group were averaged across speakers and plotted in $\mathrm{R}$ ( $\mathrm{R}$ Development Core Team, 2012). Note that the inferential statistical analyses described in the next subsection were carried out on "raw," i.e., non-normalized, tongue curves.

\section{B. Statistical analyses}

Statistical analyses were carried out in R (R Development Core Team, 2012). Linear mixed models (LMMs) were run using lmer software package (Baayen, 2008). The analyses of the $/ \mathrm{s} /-/ \int /$ contrast and of variability were carried out separately for the two vowel contexts, since these two contexts can have different effects on the two sibilant fricatives (e.g., Recasens and Espinosa, 2009). The realization of the fricative contrast as captured by the four measures (DEI, LOC $\mathrm{a}_{\mathrm{a}-\mathrm{i}}$, the fricative centroid and F2), was analysed using LMMs with Consonant and Age Group as independent variables. If a significant interaction was observed between the two factors, it would indicate developmental differences in the realization of the $/ \mathrm{s} /-/ \int /$ contrast. Uncorrelated random intercept and random slope for Speaker were included in each model. Following Reubold et al. (2010), and similarly to Zharkova et al. (2014), the denominator degrees of freedom were set at 60 , and the effect was deemed significant at the 0.05 level if the $F$ value in the analysis of variance table exceeded 7.2, and at 0.01 level if the $F$ value exceeded 8.49. Correlation analyses included comparisons of the fricative centroid at all eight intervals with the two articulatory measures. In order to ensure comparability in the correlation analyses, for each of the eight intervals, an average value of DEI and LOC $_{\mathrm{a}-\mathrm{i}}$ was taken from the two time points surrounding each interval. Correlations were also run between F2 values and both articulatory measures' values at the consonant onset and offset.

TABLE I. Mean values of the DEI [standard deviation (SD) values are in brackets] and LMM results for the main effects. Significant $F$ values are in bold font.

\begin{tabular}{|c|c|c|c|c|c|c|c|c|c|c|c|}
\hline \multicolumn{12}{|c|}{ DEI } \\
\hline \multicolumn{3}{|c|}{ Time point } & \multirow{3}{*}{$\begin{array}{c}1 \\
0.21 \\
(0.07)\end{array}$} & \multirow{3}{*}{$\begin{array}{c}2 \\
0.21 \\
(0.07)\end{array}$} & \multirow{3}{*}{$\begin{array}{c}3 \\
0.20 \\
(0.07)\end{array}$} & \multirow{3}{*}{$\begin{array}{c}4 \\
0.20 \\
(0.07)\end{array}$} & \multirow{3}{*}{$\begin{array}{c}5 \\
0.20 \\
(0.07)\end{array}$} & \multirow{3}{*}{$\begin{array}{c}6 \\
0.20 \\
(0.07)\end{array}$} & \multirow{3}{*}{$\begin{array}{c}7 \\
0.19 \\
(0.06)\end{array}$} & \multirow{3}{*}{$\begin{array}{c}8 \\
0.20 \\
(0.06)\end{array}$} & \multirow{3}{*}{$\begin{array}{c}9 \\
0.20 \\
(0.06)\end{array}$} \\
\hline /a/ & Adult & /s/ & & & & & & & & & \\
\hline & & & & & & & & & & & \\
\hline & & $/ \int /$ & 0.26 & 0.26 & 0.26 & 0.25 & 0.25 & 0.25 & 0.25 & 0.25 & 0.26 \\
\hline & & & $(0.05)$ & $(0.05)$ & $(0.06)$ & $(0.06)$ & $(0.06)$ & $(0.06)$ & $(0.06)$ & $(0.06)$ & $(0.06)$ \\
\hline & Child & /s/ & 0.24 & 0.23 & 0.23 & 0.22 & 0.22 & 0.21 & 0.22 & 0.22 & 0.22 \\
\hline & & & $(0.08)$ & $(0.08)$ & $(0.08)$ & $(0.08)$ & $(0.08)$ & $(0.08)$ & $(0.08)$ & $(0.08)$ & (0.07) \\
\hline & & $/ \int /$ & 0.29 & 0.29 & 0.29 & 0.29 & 0.29 & 0.29 & 0.29 & 0.29 & 0.30 \\
\hline & & & $(0.05)$ & $(0.05)$ & $(0.05)$ & $(0.05)$ & $(0.05)$ & $(0.05)$ & $(0.05)$ & $(0.05)$ & $(0.05)$ \\
\hline & & & 23.35 & 22.73 & 20.01 & 19.93 & 18.69 & 20.01 & 19.94 & 20.27 & 21.72 \\
\hline & & & 2.41 & 2.28 & 2.38 & 2.85 & 2.95 & 3.22 & 3.38 & 3.63 & 3.49 \\
\hline & $F, \mathrm{Cc}$ & & 0.13 & 0.05 & 0.11 & 0.21 & 0.26 & 0.49 & 0.26 & 0.44 & 0.51 \\
\hline \multirow[t]{11}{*}{ /i/ } & Adult & /s/ & 0.25 & 0.25 & 0.25 & 0.25 & 0.26 & 0.28 & 0.31 & 0.34 & 0.37 \\
\hline & & & $(0.07)$ & $(0.07)$ & $(0.07)$ & $(0.06)$ & $(0.06)$ & $(0.06)$ & $(0.06)$ & $(0.06)$ & (0.07) \\
\hline & & $/ \int /$ & 0.28 & 0.28 & 0.28 & 0.28 & 0.29 & 0.29 & 0.30 & 0.31 & 0.32 \\
\hline & & & $(0.05)$ & $(0.05)$ & $(0.06)$ & $(0.06)$ & $(0.06)$ & $(0.06)$ & $(0.07)$ & $(0.07)$ & (0.07) \\
\hline & Child & /s/ & 0.26 & 0.26 & 0.26 & 0.27 & 0.28 & 0.29 & 0.32 & 0.34 & 0.36 \\
\hline & & & $(0.08)$ & $(0.08)$ & $(0.08)$ & $(0.08)$ & $(0.07)$ & $(0.07)$ & $(0.06)$ & $(0.06)$ & $(0.06)$ \\
\hline & & $/ \int /$ & 0.30 & 0.30 & 0.30 & 0.31 & 0.31 & 0.32 & 0.33 & 0.34 & 0.35 \\
\hline & & & $(0.06)$ & $(0.06)$ & $(0.05)$ & $(0.05)$ & $(0.05)$ & $(0.05)$ & $(0.06)$ & $(0.06)$ & $(0.07)$ \\
\hline & & & 12.01 & 10.85 & 9.90 & 8.97 & 5.92 & 2.86 & 0.20 & 2.22 & 11.98 \\
\hline & & & 0.80 & 0.81 & 0.96 & 1.21 & 1.50 & 1.45 & 0.79 & 0.33 & 0.16 \\
\hline & $F, \mathrm{Cc}$ & & 0.06 & 0.06 & 0.18 & 0.20 & 0.12 & 0.40 & 0.53 & 1.84 & 5.07 \\
\hline
\end{tabular}


TABLE II. Mean values of the $\mathrm{LOC}_{\mathrm{a}-\mathrm{i}}$ (SD values are in brackets) and LMM results for the main effects. Significant $F$ values are in bold font.

\begin{tabular}{|c|c|c|c|c|c|c|c|c|c|c|c|}
\hline \multicolumn{12}{|c|}{$\mathrm{LOC}_{\mathrm{a}-\mathrm{i}}$} \\
\hline \multicolumn{3}{|c|}{ Time point } & \multirow{3}{*}{$\begin{array}{c}1 \\
0.77 \\
(0.21)\end{array}$} & \multirow{3}{*}{$\begin{array}{c}2 \\
0.77 \\
(0.21)\end{array}$} & \multirow{3}{*}{$\begin{array}{c}3 \\
0.76 \\
(0.22)\end{array}$} & \multirow{3}{*}{$\begin{array}{c}4 \\
0.76 \\
(0.22)\end{array}$} & \multirow{3}{*}{$\begin{array}{c}5 \\
0.75 \\
(0.22)\end{array}$} & \multirow{3}{*}{$\begin{array}{c}6 \\
0.76 \\
(0.23)\end{array}$} & \multirow{3}{*}{$\begin{array}{c}7 \\
0.76 \\
(0.22)\end{array}$} & \multirow{3}{*}{$\begin{array}{c}8 \\
0.77 \\
(0.21)\end{array}$} & \multirow{2}{*}{$\begin{array}{c}9 \\
0.78\end{array}$} \\
\hline /a/ & Adult & /s/ & & & & & & & & & \\
\hline & & & & & & & & & & & $(0.20)$ \\
\hline & & $/ \int /$ & 1.15 & 1.22 & 1.28 & 1.33 & 1.32 & 1.34 & 1.33 & 1.31 & 1.29 \\
\hline & & & $(0.19)$ & $(0.23)$ & $(0.26)$ & $(0.33)$ & $(0.26)$ & $(0.31)$ & $(0.26)$ & $(0.28)$ & $(0.26)$ \\
\hline & Child & /s/ & 0.83 & 0.81 & 0.80 & 0.79 & 0.79 & 0.79 & 0.80 & 0.81 & 0.83 \\
\hline & & & $(0.19)$ & $(0.20)$ & $(0.19)$ & $(0.19)$ & $(0.19)$ & $(0.19)$ & $(0.19)$ & $(0.18)$ & $(0.19)$ \\
\hline & & $/ \int /$ & 1.12 & 1.16 & 1.21 & 1.26 & 1.30 & 1.32 & 1.32 & 1.29 & 1.26 \\
\hline & & & $(0.26)$ & $(0.29)$ & $(0.31)$ & $(0.34)$ & $(0.36)$ & $(0.37)$ & $(0.36)$ & $(0.33)$ & $(0.29)$ \\
\hline & & & 108.20 & 130.26 & 136.36 & 126.84 & 141.73 & 135.96 & 159.82 & 162.91 & 198.57 \\
\hline & & & 0.13 & 0.09 & 0.12 & 0.13 & 0.17 & 0.09 & 0.23 & 0.24 & 0.42 \\
\hline & $F, \mathrm{C}$ & & 1.94 & 2.28 & 1.71 & 1.18 & 0.41 & 0.29 & 0.32 & 0.44 & 1.21 \\
\hline \multirow[t]{11}{*}{ /i/ } & Adult & /s/ & 0.82 & 0.84 & 0.83 & 0.84 & 0.86 & 0.89 & 0.99 & 1.15 & 1.30 \\
\hline & & & $(0.22)$ & $(0.23)$ & $(0.24)$ & $(0.25)$ & $(0.27)$ & $(0.28)$ & $(0.31)$ & $(0.37)$ & $(0.40)$ \\
\hline & & $/ \int /$ & 1.18 & 1.25 & 1.30 & 1.34 & 1.38 & 1.41 & 1.44 & 1.52 & 1.61 \\
\hline & & & $(0.21)$ & $(0.23)$ & $(0.24)$ & $(0.26)$ & $(0.29)$ & $(0.29)$ & $(0.30)$ & $(0.33)$ & $(0.38)$ \\
\hline & Child & /s/ & 0.86 & 0.86 & 0.87 & 0.89 & 0.91 & 0.97 & 1.06 & 1.22 & 1.39 \\
\hline & & & (0.19) & (0.19) & $(0.20)$ & $(0.21)$ & $(0.22)$ & $(0.24)$ & $(0.27)$ & $(0.37)$ & $(0.46)$ \\
\hline & & $/ \int /$ & 1.10 & 1.14 & 1.20 & 1.25 & 1.30 & 1.35 & 1.42 & 1.51 & 1.61 \\
\hline & & & $(0.21)$ & $(0.22)$ & $(0.26)$ & $(0.28)$ & $(0.30)$ & $(0.31)$ & $(0.32)$ & $(0.37)$ & $(0.44)$ \\
\hline & & & 135.05 & 158.16 & 142.44 & 135.82 & 128.44 & 111.44 & 87.76 & 41.91 & 22.68 \\
\hline & & & 0.09 & 0.09 & 0.00 & 0.02 & 0.08 & 0.09 & 0.12 & 0.04 & 0.07 \\
\hline & $F, \mathrm{C}$ & & 5.60 & 6.01 & 5.04 & 3.96 & 2.69 & 2.29 & 1.31 & 0.59 & 0.58 \\
\hline
\end{tabular}

\section{RESULTS}

Figures 3 and 4 show nine consecutive tongue contours during the two consonants for adults and preadolescents, respectively. The two age groups have rather similar patterns of tongue movement throughout the fricative. For both adults and preadolescents, tongue shapes for /s/ in the context of /a/ appear to be less bunched than those for $/ \int /$. In the context of /i/, this distinction is less noticeable.

Mean values for the articulatory measures are presented in Tables I and II, and the values for the acoustic measures can be found in Table III. $F$ values from LMMs are also presented in the tables. In all analyses including Age and Consonant as predictors, there was no significant interaction of the two factors for any measure. DEI was significantly larger for $/ \int /$ than for $/ \mathrm{s} /$ at each time point in each vowel context (meaning that the tongue was more bunched for the postalveolar fricative), except time points 5-9 in the context of /i/. At time points 5-8, the cross-consonant difference was not significant due to a progressively raising tongue dorsum for /s/, making it similar to $/ \int /$ on this aspect of tongue shape. The significant effect for DEI at the last time point of the consonant was due to this measure having a larger value for /s/ than for $/ \int /$. This pattern reflects the fact that the tongue dorsum (or, more specifically, the part of the tongue opposite the middle of line $n$ ) at this time point was more bunched for /s/, while for the postalveolar consonant the bunching occurred further towards tongue front, i.e., in the predorsum area. $\mathrm{LOC}_{\mathrm{a}-\mathrm{i}}$ was significantly larger for $/ \int /$ at all time points and vowel contexts, meaning that the front of the tongue was consistently more bunched for $/ \int /$ than for $/ \mathrm{s} /$. The centroid values were significantly higher for $/ \mathrm{s} /$ than for $/ \int /$ in both vowel contexts and at every time point. F2, both at the consonant onset and offset, was significantly greater for $/ \int /$ than for $/ \mathrm{s} /$, in both vowel contexts. For DEI, LOC $_{\mathrm{a}-\mathrm{i}}$ and the fricative centroid, there were no significant effects of Age, except for the centroid at the first interval in the context of /i/ and the last interval in the context of /a/, where on both occasions the centroid values were higher in adults than in preadolescents. For F2, Age was significant in all analyses, with preadolescent values larger than those of adults due to smaller vocal tract sizes.

Correlation results are presented in Table IV. There was a significant negative correlation between the centroid and LOC $_{\mathrm{a}-\mathrm{i}}$ throughout the consonant. A similar pattern of correlation, albeit weaker and not lasting until the end of the consonant, was observed between the centroid and DEI. F2 was positively correlated with both articulatory measures.

Table $\mathrm{V}$ presents the results of LMMs comparing variability across age group and across consonant, separately for each vowel context, and with time points pooled. The table demonstrates that the effect of age was significant only for the fricative centroid for $/ \int /$, for both vowel contexts, with larger variability in the preadolescents. Significantly more variability for /s/ than for $/ \int /$ was observed for DEI, F2, and, in the context of $/ \mathrm{i} /$, for the centroid, but only for the adults. Mean discriminability of the two fricatives was larger in adults than in children in both vowel contexts for $\mathrm{LOC}_{\mathrm{a}-\mathrm{i}}$ and the centroid, and in the context of /i/ for DEI and F2; however, Age Group was not significant in any of the analyses.

\section{DISCUSSION}

This paper presents dynamic articulatory and acoustic data on $/ \int /$ and $/ \mathrm{s} /$ produced by preadolescents and adults. The study established that phonetic characteristics 
TABLE III. Mean values of the centroid and F2 (SD values are in brackets) and LMM results for the main effects. Significant $F$ values are in bold font.

\begin{tabular}{|c|c|c|c|c|c|c|c|c|c|c|}
\hline \multicolumn{11}{|c|}{ Fricative centroid, $\mathrm{Hz}$} \\
\hline \multicolumn{3}{|c|}{ Time interval } & \multirow{3}{*}{$\begin{array}{c}1 \\
5590 \\
(1237)\end{array}$} & \multirow{3}{*}{$\begin{array}{c}2 \\
7064 \\
(997)\end{array}$} & \multirow{3}{*}{$\begin{array}{c}3 \\
7526 \\
(991)\end{array}$} & \multirow{3}{*}{$\begin{array}{c}4 \\
7707 \\
(936)\end{array}$} & \multirow{3}{*}{$\begin{array}{c}5 \\
7805 \\
(991)\end{array}$} & \multirow{3}{*}{$\begin{array}{c}6 \\
7779 \\
(981)\end{array}$} & \multirow{3}{*}{$\begin{array}{c}7 \\
7378 \\
(1056)\end{array}$} & \multirow{3}{*}{$\begin{array}{c}8 \\
5065 \\
(1363)\end{array}$} \\
\hline /a/ & Adult & /s/ & & & & & & & & \\
\hline & & & & & & & & & & \\
\hline & & $/ \int /$ & 3772 & 4092 & 4051 & 4018 & 3975 & 3929 & 3866 & 3175 \\
\hline & & & $(632)$ & $(496)$ & $(484)$ & $(482)$ & $(449)$ & $(470)$ & $(479)$ & $(689)$ \\
\hline & Child & /s/ & 5135 & 6668 & 7207 & 7471 & 7578 & 7527 & 7082 & 4817 \\
\hline & & & (1406) & (1188) & (1084) & (1093) & (1079) & $(1020)$ & (1083) & (1726) \\
\hline & & $/ \int /$ & 3197 & 3799 & 3954 & 4000 & 3928 & 3890 & 3675 & 2671 \\
\hline & & & $(760)$ & $(826)$ & $(762)$ & $(674)$ & $(607)$ & $(608)$ & $(607)$ & $(649)$ \\
\hline & $F, C$ & & 88.29 & 270.56 & 426.07 & 525.91 & 585.72 & 623.15 & 496.15 & 117.97 \\
\hline & $F$, & & 7.01 & 2.15 & 0.43 & 0.05 & 0.09 & 0.09 & 1.21 & 8.44 \\
\hline & $F$, Con & Age & 0.09 & 0.08 & 0.47 & 0.48 & 0.34 & 0.50 & 0.11 & 0.47 \\
\hline \multirow[t]{11}{*}{ /i/ } & Adult & /s/ & 6173 & 7314 & 7677 & 7840 & 7921 & 7896 & 7504 & 5682 \\
\hline & & & (1238) & (1101) & (1100) & (1078) & (1085) & (1068) & (1121) & (1171) \\
\hline & & $/ \int /$ & 4006 & 4185 & 4094 & 4100 & 4067 & 4056 & 4032 & 3560 \\
\hline & & & (492) & $(483)$ & $(478)$ & (467) & (489) & $(454)$ & $(475)$ & (488) \\
\hline & Child & /s/ & 5529 & 6753 & 7329 & 7574 & 7728 & 7710 & 7258 & 6011 \\
\hline & & & (1379) & (1211) & (1083) & (1043) & (1051) & (997) & (1034) & (974) \\
\hline & & $/ \int /$ & 3262 & 3911 & 4048 & 4101 & 4105 & 4088 & 3984 & 3368 \\
\hline & & & (801) & (797) & (735) & $(650)$ & (596) & (574) & (533) & (551) \\
\hline & $F, C$ & & 125.32 & 252.87 & 423.24 & 505.12 & $\mathbf{5 0 7 . 1 7}$ & 602.57 & 475.80 & 291.79 \\
\hline & $F$, & & 14.77 & 2.11 & 0.13 & 0.00 & 0.04 & 0.04 & 0.05 & 1.24 \\
\hline & $F$, Con & Age & 0.06 & 0.59 & 0.82 & 0.69 & 0.48 & 0.51 & 0.41 & 3.48 \\
\hline
\end{tabular}

$\mathrm{F} 2, \mathrm{~Hz}$

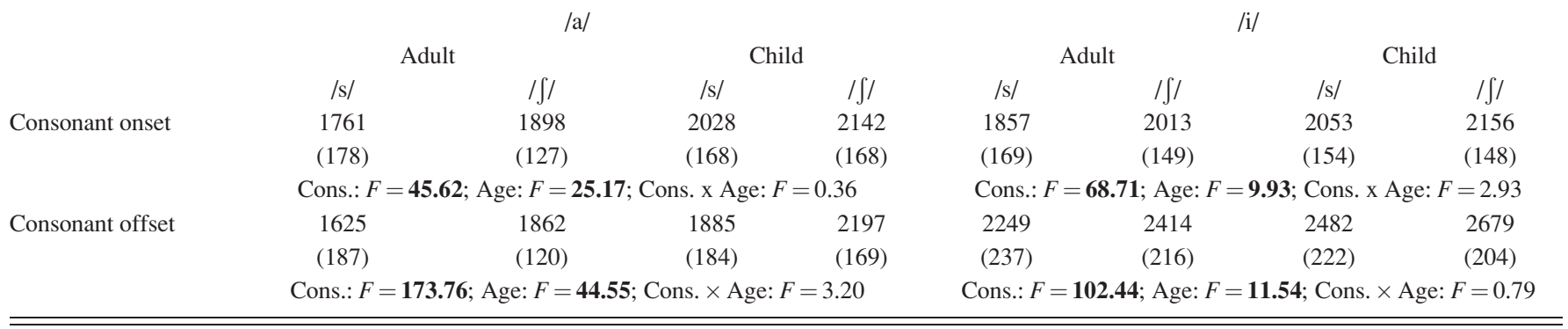

distinguishing the two sibilant fricatives are present in Scottish English speaking preadolescents. Centroid values were noticeably lower at the start and end intervals than at the other intervals, with a generally convex pattern over time [see comparable results on /s/ in Munson (2004), Iskarous et al. (2011), and Koenig et al. (2013); also, cf. results on /s/ and $/ \int /$ from Haley et al. (2010)]. Changes in tongue shape over time were also observed, particularly before the high front vowel. These findings have implications for studying fricatives in both adults and children. Specifically they suggest that measurements at several intervals during the consonant and in varied phonetic contexts are required to accurately reflect dynamic characteristics of fricative consonants.

In agreement with Romeo et al. (2013), centroids for /s/ were higher than those for $/ \mathrm{f} /$ across age groups (see also similar findings in an adult-only study reported in Haley et al., 2010). This across-fricative pattern, which has also been found in younger children (Nittrouer et al., 1989; Li et al., 2009; Holliday et al., 2015), reflects the difference in the size of the front cavity between the two fricatives. In the present study, the cross-consonant difference in both preadolescents and adults persisted throughout the duration of the consonant, as evidenced by centroid and $\mathrm{LOC}_{\mathrm{a}-\mathrm{i}}$ results. A

TABLE IV. Pearson's $r$ values for the correlation results. Bold font denotes the values that are significant at the 0.01 level, after Bonferroni adjustment.

Correlation of fricative centroid with articulatory measures

\begin{tabular}{|c|c|c|c|c|c|c|c|c|}
\hline & Int. 1 & Int. 2 & Int. 3 & Int. 4 & Int. 5 & Int. 6 & Int. 7 & Int. 8 \\
\hline DEI & -0.173 & -0.243 & -0.278 & -0.291 & -0.272 & -0.233 & -0.169 & 0.053 \\
\hline $\mathrm{LOC}_{\mathrm{a}-\mathrm{i}}$ & -0.415 & -0.501 & -0.544 & -0.572 & -0.571 & -0.538 & -0.435 & -0.144 \\
\hline \multicolumn{9}{|c|}{ Correlation of F2 with articulatory measures } \\
\hline \multicolumn{3}{|c|}{ DEI, cons. onset } & \multirow{2}{*}{\multicolumn{2}{|c|}{$\begin{array}{c}\text { LOC }_{\mathrm{a}-\mathrm{i}} \text {, cons. onset } \\
\mathbf{0 . 2 3 6}\end{array}$}} & \multicolumn{2}{|c|}{ DEI, cons. offset } & \multicolumn{2}{|c|}{$\mathrm{LOC}_{\mathrm{a}-\mathrm{i}}$, cons. offset } \\
\hline \multicolumn{2}{|l|}{0.216} & & & & \multicolumn{2}{|c|}{0.554} & \multicolumn{2}{|c|}{0.502} \\
\hline
\end{tabular}


TABLE V. Mean coefficient of variation for all measures and LMM results. Significant $F$ values are in bold font.

\begin{tabular}{|c|c|c|c|c|c|c|c|c|c|c|c|}
\hline \multicolumn{12}{|c|}{ DEI } \\
\hline & \multicolumn{3}{|c|}{ /s/, adults vs pread., CV and $F$ values } & \multicolumn{3}{|c|}{$/ \int /$, adults vs pread., $\mathrm{CV}$ and $F$ values } & \multicolumn{2}{|c|}{ LMMs on $/ \mathrm{s} / \mathrm{vs} / \int /, F$ values } & \multicolumn{3}{|c|}{ Discriminability values and $F$ values } \\
\hline & Adult & Pread. & $F$ & Adult & Pread. & $F$ & Adult & Pread. & Ad. & Pread & $F$ \\
\hline /a/ & 0.082 & 0.079 & 0.06 & 0.058 & 0.069 & 1.34 & 12.10 & 0.74 & 4.86 & 4.88 & 0.00 \\
\hline /i/ & 0.090 & 0.087 & 0.06 & 0.062 & 0.068 & 0.46 & 7.54 & 2.97 & 2.67 & 2.53 & 0.07 \\
\hline \multicolumn{12}{|c|}{$\mathrm{LOC}_{\mathrm{a}-\mathrm{i}}$} \\
\hline & \multicolumn{3}{|c|}{ /s/, adults vs pread., CV and $F$ values } & \multicolumn{3}{|c|}{$/ \int /$, adults vs pread., $\mathrm{CV}$ and $F$ values } & \multicolumn{2}{|c|}{ LMMs on /s/ vs / $\int /, F$ values } & \multicolumn{3}{|c|}{ Discriminability values and $F$ values } \\
\hline & Adult & Pread. & $F$ & Adult & Pread. & $F$ & Adult & Pread. & Ad. & Pread & $F$ \\
\hline /a/ & 0.080 & 0.086 & 0.14 & 0.064 & 0.069 & 0.23 & 1.11 & 2.14 & 8.05 & 5.92 & 3.37 \\
\hline /i/ & 0.086 & 0.098 & 0.87 & 0.077 & 0.078 & 0.00 & 0.88 & 4.23 & 5.08 & 3.66 & 4.39 \\
\hline \multicolumn{12}{|c|}{ Fricative centroid } \\
\hline & \multicolumn{3}{|c|}{ /s/, adults vs pread., $\mathrm{CV}$ and $F$ values } & \multicolumn{3}{|c|}{$/ \int /$, adults vs pread., $\mathrm{CV}$ and $F$ values } & \multicolumn{2}{|c|}{ LMMs on $/ \mathrm{s} / \mathrm{vs} / \int /, F$ values } & \multicolumn{3}{|c|}{ Discriminability values and $F$ values } \\
\hline & Adult & Pread. & $F$ & Adult & Pread. & $F$ & Adult & Pread. & Ad. & Pread & $F$ \\
\hline /a/ & 0.086 & 0.110 & 4.48 & 0.068 & 0.100 & 17.41 & 4.97 & 0.57 & 9.85 & 7.07 & 4.48 \\
\hline /i/ & 0.076 & 0.086 & 0.92 & 0.055 & 0.100 & 25.23 & 10.17 & 1.87 & 9.81 & 7.80 & 3.15 \\
\hline \multicolumn{12}{|l|}{$\mathrm{F} 2$} \\
\hline & \multicolumn{3}{|c|}{ /s/, adults vs pread., $\mathrm{CV}$ and $F$ values } & \multicolumn{3}{|c|}{$/ \int /$, adults vs pread., $\mathrm{CV}$ and $F$ values } & \multicolumn{2}{|c|}{ LMMs on $/ \mathrm{s} / \mathrm{vs} / \int /, F$ values } & \multicolumn{3}{|c|}{ Discriminability values and $F$ values } \\
\hline & Adult & Pread. & $F$ & Adult & Pread. & $F$ & Adult & Pread. & Ad. & Pread & $F$ \\
\hline /a/ & 0.050 & 0.039 & 3.72 & 0.027 & 0.032 & 1.28 & 32.96 & 2.57 & 2.98 & 3.32 & 0.30 \\
\hline /i/ & 0.038 & 0.040 & 0.11 & 0.025 & 0.029 & 2.64 & 13.16 & 3.72 & 2.53 & 2.05 & 1.29 \\
\hline
\end{tabular}

negative correlation between the centroid and $\mathrm{LOC}_{\mathrm{a}-\mathrm{i}}$ suggests that some of the difference in the front cavity size could have been brought about by differences in lingual articulation reflected in tongue shape, specifically in the location of bunching along the tongue contour, with $/ \int /$ characterized by further forward bunching along the tongue curve than /s/ throughout the consonant. As demonstrated in Figs. 3 and 4, both groups of speakers achieved this tongue shape for $/ \int /$ through raising the tongue predorsum, with a concomitant lowering of the tip/blade. In the present study it is not possible to separate any effect of the tongue shape change on the front cavity size from the influence of lip rounding or of constriction location relative to the palate, both of which would have directly affected the front cavity. However, the data from this paper suggest that some of the front cavity enlargement for the postalveolar fricative could have resulted from tip/blade lowering, which was reflected in the $/ \int /$ tongue shape captured by $\mathrm{LOC}_{\mathrm{a}-\mathrm{i}}$.

$\mathrm{LOC}_{\mathrm{a}-\mathrm{i}}$ was shown to distinguish between the two fricatives better than DEI, as the extent of tongue bunching, represented by DEI, was the same in both consonants throughout most of the second half of the fricative preceding /i/. Correlation analyses also showed that out of the two articulatory measures $\mathrm{LOC}_{\mathrm{a}-\mathrm{i}}$ was the one more consistently correlated with the fricative centroid, which is a standard acoustic measure for quantifying /s/-/ $/ /$ differences (e.g., McGowan and Nittrouer, 1988; Li et al., 2009; Romeo et al., 2013). Thus, out of the two articulatory measures, LOC $_{\mathrm{a}-\mathrm{i}}$ would be more useful in further articulatory studies using midsagittal tongue contours for characterising fricative production in adults and children.

The second formant captured the difference between the two fricatives in the length of the cavity behind the constriction at the start and end of the fricative (cf. comparable findings in Nittrouer et al., 1989; 1996), with the tongue-to-palate constriction for $/ \int /$ being further back in the oral cavity than for /s/. Results from the articulatory measures at the consonant onset and offset, as well as the correlation analyses, suggest that the tongue might have contributed to creating this difference through the extent and relative location of its most bunched part, with larger bunching for $/ \int /$ in the tongue dorsum and predorsum area contributing to reducing the back cavity. This interpretation ties in with an observation about the difference in F2 between /s/ and / //, made in an acoustic-only study of fricatives by McGowan and Nittrouer (1988): "the configuration of the tongue body contributes to this difference as well in that the tongue body is higher and more fronted for $/ \int /$ than for /s/" (p. 232).

The effect of age on the fricative production was mostly not significant, with exceptions observed for the centroid, close to the fricative-vowel boundaries. Age-related differences were brought about by lower values in preadolescents, possibly due to a more retracted constriction, and/or to a wider constriction with a consequent larger drop in highfrequency energy at the periphery of the consonant in preadolescents than in adults (cf. Shadle et al., 2014). These differences between the two groups suggest the need to include teenage participants in future developmental studies addressing the dynamics of sibilant fricative production (cf. Romeo et al., 2013, where no age-related differences in centroid values were observed at the middle portion of the consonant, but additional analyses of /s/-/ / / discriminability suggested that some age-related changes towards adult-like categories must take place after the age of 14 years old). The present study analysed midsagittal tongue images, but did not include coronal scans (cf. Stone et al., 1992, where coronal ultrasound images were used to show across-fricative differences in grooving for an adult speaker). It would be interesting to include coronal tongue images in future studies of 
child and adult fricative production, since coordinating the tongue sides with the tongue midline to form a midsagittal groove requires complex muscle activity, and developmental differences could be predicted.

Significantly greater variability in preadolescents than in adults was only observed for one measure, namely, the fricative centroid, and only for $/ \int /$ and not for $/ \mathrm{s} /$. The fact that larger-than-adult variability for the preadolescents was not found for any of the two articulatory measures agrees with the findings reported by Zharkova et al. (2014), who used the same dataset as in the current study, and found that variability in absolute tongue position in the mouth across repetitions was comparable in adults and preadolescents. The difference in centroid variability between the two groups of speakers might have been due to the fact that $/ \int /$ has "stricter production requirements" [Recasens and Mira (2013), p. 320] than /s/, since the tongue dorsum, involved in the tongue-to-palate constriction for $/ \int /$, is a larger articulator than the blade, and is therefore characterized by more inertia, making / / / highly resistant to coarticulatory influence from neighbouring sounds (Recasens and Espinosa, 2009). Consequently, it could be that preadolescents do not yet follow these stricter requirements as consistently as adults, and the centroid variability reflects somewhat inconsistent constriction locations across repetitions of $/ \int /$. An added complication in the production of $/ \int /$ is the requirement for the tongue tip to have no contact with the lower teeth (Perkell et al., 1979), in order to create a sublingual space (Nittrouer, 1995, explained disproportionately higher / $/$ / centroid values for 3-to-7-year-old children than for adults by suggesting that the children may have had contact between the tongue and the mandibular arch; cf. also Nicholson et al., 2015). Alternative explanations are related to the nature of the data reported here. As mentioned above, the study did not present information on the sides of the tongue, which are important for the production of fricatives (Stone et al., 1992; Narayanan et al., 1995); nor was there information on the lips, which are particularly relevant for $/ \int /$. Larger variability in preadolescents might have been observed on both lip movement and that of the tongue margins. Also, the tongue data were not presented within the jaw frame (cf. Mooshammer et al., 2007; Iskarous et al., 2011). It is possible that the greater centroid variability in preadolescents for $/ \int /$ reflected larger variability in the coordination of the tongue and the jaw in preadolescents (cf. Cheng et al., 2007), combined with the requirement for lip rounding for the postalveolar. It is interesting that the larger variability of $/ \int /$ centroid in the preadolescents did not lead to age-related differences in the overall discriminability of the two fricatives. On the centroid, as well as on the other measures, discriminability was not significantly different between the two age groups, albeit absolute values were mostly smaller in the preadolescents.

As for cross-consonant differences in variability, in the preadolescents, like in the adults, absolute differences in mean coefficients of variation for all four measures were larger for $/ \mathrm{s} /$ than for $/ \int /$. However, while the adults showed significantly more variability for $/ \mathrm{s} /$ than for $/ \int /$ on the extent of tongue bunching and on the acoustic measures, none of these differences reached significance in the preadolescents.
Perhaps additional articulatory details not reported in this study would have made it possible to observe this pattern in the preadolescent group. It is also possible that the differential centroid variability of /s/ and / $/$ / was not present in the preadolescents due to their relatively large centroid variability for $/ \int /$, discussed above.

Previous studies of sibilant fricatives in children, adolescents and adults have reported gender differences, with consistently lower fricative centroid values in males than in females [for children and adolescents, see Flipsen et al. (1999) and Koenig et al. (2013); for comparisons with adults, see Romeo et al. (2013); cf. also adult-only data in Jongman et al. (2000) and Weirich and Simpson (2015)]. In the present study, gender was not included in fricative centroid analyses reported above. However, when these analyses were rerun with gender as an additional fixed factor, there were no significant interactions of this factor with any other factors; female centroid values were significantly higher than male values between the 4th and the 7 th intervals for $/ \mathrm{a} /$, and between the 5 th and the 7 th intervals for $/ \mathrm{i} /$. There is a possibility that during those intervals the adult mean centroid would have been higher than the preadolescent mean centroid because there were more female speakers in the adult than in the preadolescent group. However, when separate LMMs were carried out within each gender group on those intervals, the results for both groups were the same as the results of the original models with the two genders pooled. In the abovementioned study, Romeo et al. (2013) found that 9-10-year-old girls had much lower centroid values for /s/ than 11-12-year-old and 13-14-year-old girls, while no such differences were reported for $/ \int /$. In the present study, only one girl was aged under 11 years old (she was 10 years, 6 months), and her centroid values for /s/ were within one standard deviation of all the other girls' group values.

This study did not focus on vowel-related effects, although some qualitative observations can be made from Tables I-III. All mean group values for DEI, centroid and F2 for each consonant and at each measurement point were higher in the context of /i/ than in the context of /a/, in both groups of speakers (cf. Shadle and Mair, 1996; Iskarous et al., 2011; Reidy, 2015). Mean group values for $\mathrm{LOC}_{\mathrm{a}-\mathrm{i}}$ followed the same pattern, with the exception of the first half of $/ \int /$ for the preadolescents (cf. Zharkova et al., 2014). Consonant-specific coarticulatory effects from contrasting vowels have been demonstrated in a number of studies to follow different developmental patterns depending on articulatory requirements for the target consonant production (e.g., Sussman et al., 1999; Katz and Bharadwaj, 2001; Reidy, 2015). Studying these patterns makes it possible to better understand the typical development of speech motor control. Future work will include analysing fricatives in contrasting vowel contexts in several age groups throughout childhood until adolescence, using combined information from ultrasound tongue imaging and the acoustic signal.

This study showed a consistent relationship between tongue shape and cavity size in the production of sibilant fricatives in preadolescents and adults. The study also identified an articulatory measure, $\mathrm{LOC}_{\mathrm{a}-\mathrm{i}}$, which is able, similarly to the fricative centroid, to distinguish between $/ \mathrm{s} /$ and $/ \int /$ throughout 
the consonant duration, in different segmental contexts. This measure may be useful in future studies of fricative differentiation in young children and in disordered speech (cf. Bernhardt et al., 2015; Holliday et al., 2015; Neumeyer et al., 2015), where direct information on tongue shape may indicate the nature of immaturities in fricative production.

\section{ACKNOWLEDGMENTS}

The author is grateful to Bill Hardcastle, Jim Scobbie, and Daniel Recasens for comments on an earlier draft of this manuscript, to Robin Lickley for his help with the formant measurement reliability analysis, and to Valerie Hazan and Sonia Granlund for sharing the scripts for spectral moment analysis. This research was supported by two grants from the Economic and Social Research Council (Grant Nos. RES000-22-4075 and ES/K002597/1).

\footnotetext{
1"Dynamic" in this context refers to measurements carried out during the time course of the fricative.

${ }^{2}$ In one adult speaker and two preadolescent speakers, the shadow of the hyoid bone was not consistently visible throughout most of each recording, and consequently the very end of the bright white line for the tongue just above the hyoid bone shadow was consistently beyond the ultrasound field of view; in another adult speaker and two preadolescent speakers, this was the case only for some consonant-vowel targets. However when linear mixed models on DEI and $\mathrm{LOC}_{\mathrm{a}-\mathrm{i}}$ were rerun excluding those tokens, the results did not change.
}

Articulate Instruments Ltd. (2012). Articulate Assistant Advanced Ultrasound Module User Guide: Version 2.14 (Articulate Instruments Ltd., Edinburgh, UK).

Baayen, R. H. (2008). Analyzing Linguistic Data. A Practical Introduction to Statistics using $R$ (Cambridge University Press, Cambridge), pp. 241-302.

Bernhardt, B. M., Másdóttir, T., Stemberger, J. P., Leonhardt, L., and Hansson, G. Ó. (2015). "Fricative acquisition in English- and Icelandicspeaking preschoolers with protracted phonological development," Clin. Ling. Phon. 29, 642-665.

Blacklock, O. (2004). "Characteristics of variation in production of normal and disordered fricatives, using reduced-variance spectral methods," $\mathrm{Ph} . \mathrm{D}$. thesis, School of Electronics and Computer Science, University of Southampton, Southampton, UK.

Boersma, P., and Weenink, D. (2013). "Praat: Doing phonetics by computer" [Computer program], version 5.3.52, http://www.praat.org/ (Last viewed 6/12/13).

Cheng, H. Y., Murdoch, B. E., Goozée, J. V., and Scott, D. (2007). "Physiologic development of tongue-jaw coordination from childhood to adulthood," J. Speech Lang. Hear. Res. 50, 352-360.

Flipsen, P., Shriberg, L., Weismer, G., Karlsson, H., and McSweeny, J. (1999). "Acoustic characteristics of /s/ in adolescents," J. Speech Lang. Hear. Res. 42, 663-677.

Haley, K. L., Seelinger, E., Mandulak, K. C., and Zajac, D. J. (2010). "Evaluating the spectral distinction between sibilant fricatives through a speaker-centered approach," J. Phon. 38, 548-554.

Holliday, J. J., Reidy, P. F., Beckman, M. E., and Edwards, J. (2015). "Quantifying the robustness of the English sibilant fricative contrast in children,” J. Speech Lang. Hear. Res. 58, 622-637.

Iskarous, K., Shadle, C. H., and Proctor, M. I. (2011). "Articulatory-acoustic kinematics: The production of American English /s/," J. Acoust. Soc. Am. 129, 944-954.

Jongman, A., Wayland, R., and Wong, S. (2000). "Acoustic characteristics of English fricatives," J. Acoust. Soc. Am. 108, 1252-1263.

Katz, W., and Bharadwaj, S. (2001). "Coarticulation in fricative-vowel syllables produced by children and adults: A preliminary report," Clin. Ling. Phon. 15, 139-143.

Koenig, L. L., Lucero, J. C., and Perlman, E. (2008). "Speech production variability in fricatives of children and adults: Results of functional data analysis," J. Acoust. Soc. Am. 124, 3158-3170.
Koenig, L. L., Shadle, C. H., Preston, J. L., and Mooshammer, C. R. (2013). "Toward improved spectral measures of /s/: Results from adolescents," J. Speech Lang. Hear. Res. 56, 1175-1189.

Lee, S., Potamianos, A., and Narayanan, S. (1999). “Acoustics of children's speech: Developmental changes of temporal and spectral parameters," J. Acoust. Soc. Am. 105, 1455-1468.

Li, F., Edwards, J., and Beckman, M. E. (2009). "Contrast and covert contrast: The phonetic development of voiceless sibilant fricatives in English and Japanese toddlers," J. Phon. 37, 111-124.

McGowan, R. S., and Nittrouer, S. (1988). "Differences in fricative production between children and adults: Evidence from an acoustic analysis of /s/ and /s/," J. Acoust. Soc. Am. 83, 229-236.

Mooshammer, C., Hoole, P., and Geumann, A. (2007). "Jaw and order," Lang. Speech 50, 145-176.

Munson, B. (2004). "Variability in /s/ production in children and adults: Evidence from dynamic measures of spectral mean," J. Speech Lang. Hear. Res. 47, 58-69.

Narayanan, S., Alwan, A. A., and Haker, K. (1995). "An articulatory study of fricative consonants using magnetic resonance imaging," J. Acoust. Soc. Am. 98, 1325-1347.

Neumeyer, V., Schiel, F., and Hoole, P. (2015). "Speech of cochlear implant patients: An acoustic analysis of sibilant production," in Proceedings of the 18th International Congress of Phonetic Sciences, edited by the Scottish Consortium for ICPhS 2015, the University of Glasgow, Glasgow, UK, paper number 178, pp. 1-5.

Nicholson, H., Munson, B., Reidy, P., and Edwards, J. (2015). "Effects of age and vocabulary size on production accuracy and acoustic differentiation of young children's sibilant fricatives," in Proceedings of the 18th International Congress of Phonetic Sciences, edited by the Scottish Consortium for ICPhS 2015, the University of Glasgow, Glasgow, UK, paper number 831 , pp. $1-5$.

Nittrouer, S. (1995). "Children learn separate aspects of speech production at different rates: Evidence from spectral moments," J. Acoust. Soc. Am. 97, 520-530.

Nittrouer, S., Studdert-Kennedy, M., and McGowan, R. S. (1989). "The emergence of phonetic segments: Evidence from the spectral structure of fricative-vowel syllables spoken by children and adults," J. Speech Hear. Res. 32, 120-132.

Nittrouer, S., Studdert-Kennedy, M., and Neely, S. T. (1996). "How children learn to organize their speech gestures: Further evidence from fricativevowel syllables," J. Speech Hear. Res. 39, 379-389.

Perkell, J. S., Boyce, S. E., and Stevens, K. N. (1979). "Articulatory and acoustic correlates of the [s-š] distinction,” J. Acoust. Soc. Am. 65, S24.

$\mathrm{R}$ Development Core Team. (2012). "R: A language and environment for statistical computing," R Foundation for Statistical Computing, Vienna, http://www.R-project.org (Last viewed 6/1/12).

Recasens, D., and Espinosa, A. (2009). "An articulatory investigation of lingual coarticulatory resistance and aggressiveness for consonants and vowels in Catalan," J. Acoust. Soc. Am. 125, 2288-2298.

Recasens, D., and Mira, M. (2013). "An articulatory and acoustic study of the fricative clusters /s $\int /$ and / $\int \mathrm{s} /$ in Catalan," Phonetica 70, 298-322.

Reidy, P. F. (2013). "An introduction to random processes for the spectral analysis of speech data," Ohio State Univ. Work. Papers Ling. 60, 67-116.

Reidy, P. F. (2015). "The spectral dynamics of voiceless sibilant fricatives in English and Japanese," Ph.D. thesis, Graduate Program in Linguistics, The Ohio State University, Columbus, OH.

Reubold, U., Harrington, J., and Kleber, F. (2010). "Vocal aging effects on F0 and the first formant: A longitudinal analysis in adult speakers," Speech Commun. 52, 638-651.

Romeo, R., Hazan, V., and Pettinato, M. (2013). "Developmental and gender-related trends of intra-talker variability in consonant production," J. Acoust. Soc. Am. 134, 3781-3792.

Shadle, C. H., Koenig, L. L., and Preston, J. L. (2014). “Acoustic characterization of /s/ spectra of adolescents: Moving beyond moments," Proc. Mtg. Acoust. 12, 1-20.

Shadle, C. H., and Mair, S. J. (1996). "Quantifying spectral characteristics of fricatives," in Proceedings of the International Conference on Spoken Language Processing (ICSLP), Philadelphia, pp. 1521-1524.

Stone, M., Faber, A., Raphael, L. J., and Shawker, T. H. (1992). "Cross-sectional tongue shape and linguopalatal contact patterns in [s], [ $]$, and [1]," J. Phon. 20, 253-270.

Sussman, H. M., Duder, C., Dalston, E., and Cacciatore, A. (1999). "An acoustic analysis of the development of CV coarticulation: A case study," J. Speech Lang. Hear. Res. 42, 1080-1096. 
Walsh, B., and Smith, A. (2002). "Articulatory movements in adolescents: Evidence for protracted development of speech motor control processes," J. Speech Lang. Hear. Res. 45, 1119-1133.

Weirich, M., and Simpson, A. (2015). "Gender-specific differences in sibilant contrast realizations in English and German," in Proceedings of the 18th International Congress of Phonetic Sciences, edited by the Scottish Consortium for ICPhS 2015, the University of Glasgow, Glasgow, UK, paper number 261 , pp. $1-4$.

Zharkova, N. (2013a). "Using ultrasound to quantify tongue shape and movement characteristics," Cleft Palate-Craniofacial J. 50, 76-81.
Zharkova, N. (2013b). "A normative-speaker validation study of two indices developed to quantify tongue dorsum activity from midsagittal tongue shapes," Clin. Ling. Phon. 27, 484-496.

Zharkova, N., Gibbon, F. E., and Hardcastle, W. J. (2015). "Quantifying lingual coarticulation using ultrasound imaging data collected with and without head stabilisation," Clin. Ling. Phon. 29, 249-265.

Zharkova, N., Hewlett, N., Hardcastle, W. J., and Lickley, R. J. (2014). "Spatial and temporal lingual coarticulation and motor control in preadolescents," J. Speech Lang. Hear. Res. 57, 374-388. 